\title{
Gobernanza hídrica como securitización socioambiental en la subcuenca La Sabana-Tres Palos, Acapulco
}

\author{
Erick Alfonso Galán Castro, \\ América Libertad Rodríguez Herrera, José Luis Rosas-Acevedo
}

\begin{abstract}
Resumen: Este artículo analiza tres tipos de gobernanza del agua en la subcuenca del Río La Sabana-Laguna de tres Palos desde la perspectiva de la gubernamentalidad de Michel Foucault. Estos procesos-el Consejo de Administración del Organismo Operador de Agua metropolitano denominado Comisión de Agua Potable y Alcantarillado del Municipio de Acapulco (CAPAMA), el Consejo de Cuenca del Río La SabanaLaguna de Tres Palos, y una experiencia de gestión comunitaria de agua en la localidad de Kilómetro 30, del mismo municipio de Acapulco-son observados mediante la pregunta: ¿Cómo se gubernamentaliza la relación entre ciudadanos y funcionarios para la gestión del agua en esa región? Los actores perciben una mayor tendencia al control político que a la democratización en la toma de decisiones.
\end{abstract}

Palabras clave: ciudadanía, gubernamentalidad, ilegalidad, recursos hídricos, territorios de cuenca

El manejo integral de cuencas en México es un tema largamente discutido desde la literatura académica, y que ha supuesto también una serie de políticas ambientales que han buscado romper el paradigma del uso extensivo del agua para el desarrollo del país (Aboites, 2004; Perevochtchikova \& Arellano, 2009). La puesta en marcha de experiencias de gobernanza ambiental en México, y su relativa funcionalidad que puede verse en su supervivencia a pesar de la desconfianza ciudadana, son de especial relevancia en esta coyuntura histórica. La opinión pública en México debate entre la necesidad de mantener los mecanismos de cogestión ciudadana, por un lado, o fortalecer un Estado que puede ser transformado desde su interior mediante la voluntad de los funcionarios públicos y el castigo a la corrupción, por el otro. 
Los casos de los consejos de administración de los Organismos Operadores de Agua (OOs, en lo sucesivo) descentralizados, los Consejos de Cuenca y los Comités Comunitarios encierran, cada uno, complejidades muy diversas. Es posible pensar que no hay interconexión entre estas tres formas de cogestión de recursos hídricos, pero para el caso de la subcuenca del Rio La Sabana-Laguna de Tres Palos en Acapulco, Guerrero, son relevantes por poseer problemáticas comunes: la convergencia de actores civiles, gubernamentales y de habitantes de las cercanías del río que generan mecanismos de toma de decisión conjunta sobre el recurso hídrico en un escenario históricamente caracterizado por la baja confianza en las instituciones gubernamentales, y la alta conflictividad expresada en disputas armadas (entre caciques locales, entre grupos criminales, etc.). Carlos Illades (2010) nos habla de que el estado de Guerrero se ha constituido históricamente en la búsqueda del control gubernamental de territorios prácticamente incomunicados, por parte de diversos caudillos políticos locales, detonando en muchas ocasiones conflictos armados y violencia. Afirmamos que estas tres experiencias de gobernanza hídrica en la subcuenca, a pesar de las mencionadas diferencias técnicas y legales, son producto de este proceso histórico, y esta situación cobra especial relevancia en medio de la crítica del actual gobierno federal al desempeño de los mecanismos cogestivos.

La subcuenca del Rio La Sabana-Laguna de Tres Palos agrupa más de 60 poblaciones localizadas entre la comunidad de La Providencia y la zona metropolitana de la ciudad de Acapulco, Guerrero, estado ubicado al sur de México (González et al., 2012, p. 14). El aumento de la población en los últimos 20 años en las colonias asentadas cerca de la subcuenca del Río La Sabana-Laguna de Tres Palos, junto con otros problemas como el aumento de actividades económicas no formales, las afectaciones hidrometeorológicas producto del cambio climático y la falta de atención estatal sobre el tratamiento de aguas residuales en la zona, han generado no solo una mala calidad del recurso hídrico, sino también otros problemas de carácter social (asentamientos irregulares que descargan aguas residuales de uso doméstico, manejo irregular de Residuos Sólidos Urbanos), ambiental (degradación del suelo, baja calidad del agua para su consumo humano, pérdida masiva de cubierta vegetal, erosión), y económico (pérdida de recursos pesqueros y daños considerables en la zona turística de la Laguna de Tres Palos) (González et al., 2012, p. 24; Saldaña \& Pérez, 2015, p. 2; Sánchez Infante, 2018, p. 18).

A través de otros estudios (Donoso, 2017; Triana, 2017), podemos ver cómo en colonias cercanas a la zona baja de la subcuenca se han presentado eventos de violencia criminal que limitan la calidad de vida en esta región. Producto de estas problemáticas, emerge una notoria desconfianza hacia las autoridades estatales de los tres órdenes de gobierno. 
El objetivo del presente trabajo es analizar tres experiencias de gobernanza del agua dentro de la subcuenca del Río La Sabana-Laguna de Tres Palos, desde la perspectiva analítica de la gubernamentalidad de Michel Foucault. A saber: el Consejo de Administración del Organismo Operador de Agua denominado Comisión de Agua Potable y Alcantarillado del Municipio de Acapulco (CAPAMA), el Consejo de Cuenca del Río La SabanaLaguna de Tres Palos, y una experiencia de gestión comunitaria de agua en la localidad de Kilómetro 30, del mismo municipio de Acapulco. ${ }^{1}$ La pregunta central de investigación es, ¿qué aportes ofrecen los planteamientos teóricos sobre el control y el poder de Michel Foucault y Achille Mbembé para comprender la relación entre actores ciudadanos y gubernamentales en la gestión de recursos hídricos dentro de la subcuenca Río La SabanaLaguna de Tres Palos? Sostenemos que los problemas estructurales a nivel de la relación sociedad-Estado en Acapulco, dan como resultado la puesta en marcha de un mecanismo de "seguridad socioambiental", ${ }^{2}$ que supone una "biopolítica socioambiental": implementación de mecanismos legales, institucionales y procedimentales que permiten la participación de los ciudadanos en la gestión de los recursos hídricos; y una "necropolítica socioambiental": abandono selectivo de sectores poblacionales históricamente olvidados, control mediante "gobierno privado indirecto" e implementación de un estado de excepción donde la eliminación impune de los grupos marginados es una posibilidad real.

Luego de esta introducción presentaremos las categorías de análisis empleadas en este estudio, posteriormente detallaremos la metodología mixta de investigación en la cual combinamos la reflexión teórica con datos empíricos, seguida de la exposición de resultados de nuestro análisis a partir de la reconstrucción de dos procesos de construcción de gobernanza hídrica en el caso de estudio: la biopolítica y la necropolítica. Al final discutiremos algunas conclusiones a la luz de la información analizada.

\section{Gobernanza, gubernamentalidad y seguridad socioambiental}

Consideramos que el análisis de la gubernamentalidad es pertinente para interpretar la construcción del poder político en un contexto neoliberal, ${ }^{3}$ y las consecuencias que ha tenido dicho contexto en constitución de relaciones existentes entre gobernantes y gobernados. La perspectiva seleccionada incluye un análisis complejo de las relaciones de poder basadas en el manejo de recursos en una región determinada, analizando críticamente las consecuencias de la implementación neoliberal en contextos de precariedad institucional. Este enfoque considera la crisis neoliberal como generadora de control social de las poblaciones, a pesar de la existencia 
de teorías que analizan el mismo problema como las que rescatan el concepto de "sociedad civil" (Cohen \& Arato, 2001), "gobernanza democrática" (Aguilar Villanueva, 2016), o las que, criticando al Estado Liberal, se centran en el comunitarismo como forma de acción política.

La gobernanza parte de la inclusión de los actores ciudadanos para cogestionar el poder y generar espacios de inclusión en la toma de decisiones, en un marco de insuficiencia estatal para administrar el poder (Aguilar Villanueva, 2016). La incidencia gubernamental sobre el recurso en disputa y la participación ciudadana, son los ejes que definen el equilibrio que el proceso de gobernanza deberá de mantener.

La "gobernanza del agua", en ese sentido, ha sido una tendencia global desde los años 80 del siglo pasado en el marco de la puesta en marcha del proyecto neoliberal por cogestionar los recursos hídricos entre actores estatales y ciudadanos. El enfoque busca planear, implementar y evaluar las políticas ambientales sobre el agua, además de crear espacios institucionales de contrapeso al Estado, y dar a los ciudadanos la capacidad de desarrollar sus propias estrategias de gestión con la menor participación estatal posible (Castro et al., 2004; Mussetta, 2009; Pacheco-Vega, 2015).

El análisis de la gobernanza del agua como forma de gubernamentalidad pone de relieve el carácter dinámico y relacional de la cogestión de los recursos hídricos. Según Foucault, la gubernamentalidad supone también la relación entre quienes ejercen el poder del Estado y las poblaciones a quienes busca controlar. Este vínculo no es conflictivo ni violento, solo pone sutilmente en práctica el control a las poblaciones como producto de la modernidad. (Foucault, 2018, p. 136).

El centro de la reflexión general de la obra de Foucault es el ejercicio del poder, sus aristas, su genealogía y sus procesos de manifestación. El poder y la dominación están íntimamente ligados, aunque no están circunscritos a la influencia ejercida de parte de un ego a un alter: poder y dominación son una fuerza disputada constantemente. Quien ejerce el poder en un momento, lo hace otorgando un espacio de poder a quien intenta dominar, evitando un juego de suma cero. En este sentido, los gobiernos no solo controlan y reprimen a las poblaciones, también otorgan reconocimiento, autonomía limitada, presencia jurídica, para no exceder los límites de la capacidad gubernamental de dominio (Foucault, 2018). El otorgamiento de espacios institucionales para la participación ante la aparente incapacidad estatal, como asume la teoría clásica de la gobernanza, sería una forma de "gubernamentalizar" la gestión de recursos hídricos.

El gobierno de las poblaciones presenta retos ante las condiciones de escasez del agua, estado que afecta a numerosos actores y no se resuelve con la simple distribución total del recurso, sino que requiere la mediación gubernamental entre las capacidades institucionales para re- 
solver el problema, la necesidad de generar controles hacia la población, al mismo tiempo de generar confianza entre ésta. La formulación de políticas socioambientales, proceso político en la implementación del neoliberalismo, dio como resultado la "seguridad socioambiental", definida como el conjunto de dispositivos generados desde la gubernamentalidad para gestionar la escasez de recursos naturales en contextos de violencia y criminalidad. Esto supone dos vías posibles de gestión: una que está orientada a garantizar al mismo tiempo la sobrevivencia y la gestión sustentable de recursos naturales, una "biopolítica socioambiental"; y otra que supondría no solo el manejo extensivo y no circular de recursos naturales, sino el abandono y la eliminación manifiesta de poblaciones, una "necropolítica socioambiental".

Este proceso considera complejas tramas de decisión política que giran en torno a dos posibilidades: la de otorgar reconocimiento a un grupo de la población en espacios determinados, beneficiándole con el acceso a los recursos en un escenario de escasez, "biopolítica" según Foucault (2007, p. 43); o la generación de políticas excluyentes, que olvidan y abandonan a otras poblaciones de manera deliberada (o no), y que devienen en una forma de dejar morir, lo que Mbembé (2011) denomina "necropolítica".

Caben aquí dos precisiones sobre el concepto de "necropolítica". La primera relacionada con su procedencia: Mbembé formula dicho concepto como una forma de abonar a un debate en el que Foucault no alcanzó a aportar: el papel de los regímenes no occidentales en la preservación del balance entre la vida y la muerte (Falomir Archambault, 2011, p. 14). Es decir, el concepto de "necropolítica" o el más genérico de "necropoder" no son un aporte directo de Foucault, quien solamente logró formular el concepto de "tanatopolítica" como la forma más cercana al necropoder: el "reverso" de la biopolítica que genera muerte (Foucault, 2013). La segunda tiene que ver con sus alcances: la necropolítica no se define esencialmente por la acción directa del gobierno para matar a otros, aunque su manifestación más grotesca sea el genocidio, sino su capacidad para "dejar morir" (Mbembé, 2011, pp. 19-20). Así, un gobierno necropolítico no solo mata a sus oponentes, sino que genera las condiciones para lograr su control a partir de su desprotección, de "dejar a su suerte" a la población que no puede controlar.

Todos los actores involucrados en el juego de la gobernanza-los gobernantes, las poblaciones reconocidas y las poblaciones olvidadas-juegan este juego de poder y no siempre lo hacen bajo la lógica imperante del Estado de Derecho. Para lograr la resolución del problema fundante, la escasez del agua en sí, todos ellos pueden recurrir a medios legales o extralegales. La disputa por la sobrevivencia en un contexto de gubernamentalidad también pasa por las negociaciones y disputa lo que Veena 
Das y Deborah Poole (2008) denominan "los márgenes del Estado". Este concepto supone la existencia de "legalidades paralelas" que, aunque suponen la posibilidad de recurrir al Estado de Derecho para zanjar conflictos, también puede recurrirse a la corrupción, la violencia y la impunidad para generar control en contextos de escasez.

La gobernanza hídrica como necropolítica supone una forma de securitización socioambiental basada en los siguientes aspectos: (1) la excepcionalidad expresada en la apariencia jurídica de participación ciudadana e incidencia que no es garantizada en el procedimiento directo de la gestión; (2) la emergencia del gobierno privado indirecto mediante legalidades paralelas, corrupción, impunidad, y presencia selectiva del Estado; y (3) el "dejar morir", evidente en la relación entre exclusión de los actores en la toma de decisiones y la emergencia de "corredores de riesgo socioambiental". En estos corredores no solo el acceso a recursos hídricos de calidad es difícil, sino que también se han tornado espacios de muerte en los que la desigualdad, la amenaza directa a defensores del territorio, y la violencia son escenario cotidiano.

\section{Metodología}

La subcuenca del Río La Sabana-Laguna de Tres Palos, en el estado de Guerrero, México, tiene una gran peculiaridad socioambiental en la región. El aumento de las actividades turísticas y la transformación urbana de la ciudad de Acapulco, ha impactado en la configuración de la subcuenca. En ella se construyeron zonas habitacionales en las cuales el gobierno buscó refugiar a los habitantes de las colonias ubicadas al norte de la ciudad, que concentraba muchos migrantes de distintas regiones del estado de Guerrero, especialmente de la costa chica. Estas colonias, Ciudad Renacimiento y El Coloso, han sido consideradas-desde su inauguración-áreas de alto riesgo por el nivel de criminalidad y violencia (Donoso, 2017; Jiménez Badillo, 2017; Triana, 2016). La construcción de estos espacios ha supuesto una mayor contaminación de las aguas del Rio La Sabana y de la Laguna de Tres Palos, por las constantes descargas de aguas residuales domésticas, sea por el drenaje público o por desagües (Sánchez Infante, 2018). El Río mide aproximadamente $57 \mathrm{~km}$ hasta llegar a la Laguna de Tres Palos, de una longitud máxima de $16 \mathrm{~km}$ y una anchura mayor de seis km (Sánchez Infante \& Rosas, 2017, p 195).

Para resolver la pregunta de investigación hemos diseñado un acercamiento a la problemática desde un enfoque cualitativo que toma como unidad de análisis las experiencias de los actores como parte del entramado que construye la gestión del agua en la subcuenca Rio La Sabana-Laguna 


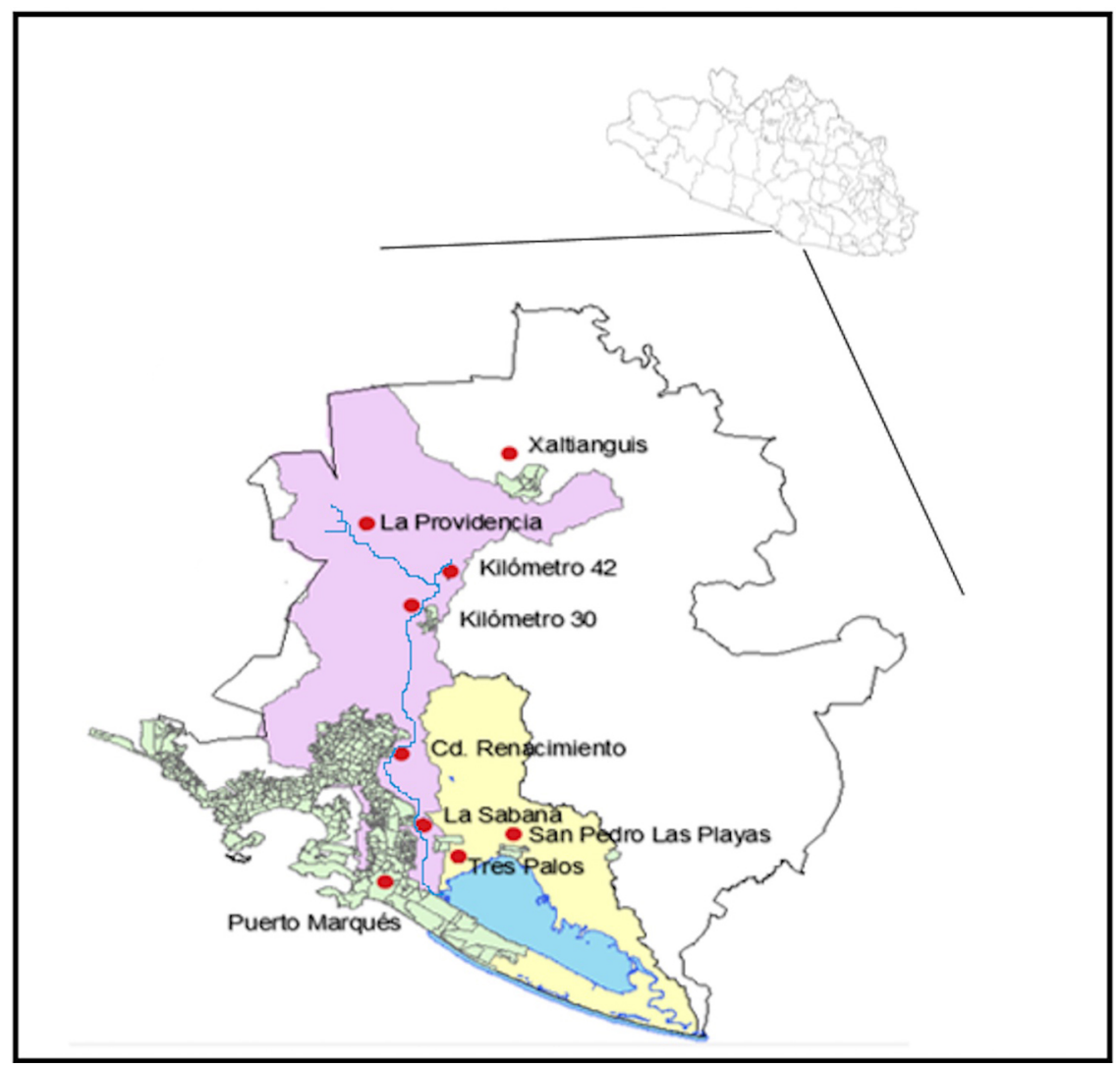

Figura 1 - Mapa de la Subcuenca Rio la Sabana-Laguna de Tres Palos Fuente: Elaboración del Mtro. Raul Torres Rico

de Tres Palos. Elaboramos 30 entrevistas a profundidad a tres grupos de actores: actores gubernamentales (funcionarios públicos relacionados a la gestión del agua en los tres órdenes de gobierno), actores de la esfera civil (académicos que conforman el Consejo de Cuenca, actores involucrados a Organizaciones de la Sociedad Civil involucrados en temas ambientalistas), y actores comunitarios de la gestión del agua en la localidad de Kilómetro 30. El análisis realizado mediante Atlas.ti a partir de una codificación mixta (códigos cerrados y abiertos), derivó en la recuperación de un fichero de 446 citas, la emergencia de 68 códigos de los cuales 16 fueron cerrados y 52 abiertos. Dicho análisis reconstruyó la relación existente entre la implementación de mecanismos de gobernanza hídrica con conceptos como la seguridad socioambiental. Ante la dificultad de mostrar todos los extractos textuales recuperados, al inicio de la sección de resultados mostramos el mapa textual derivado del análisis triangulado de 
dichos referentes empíricos, los cuales hemos incorporado a una reflexión teórica que permita articular argumentos conceptuales consolidados con evidencia textual.

Hemos procedido a resguardar la identidad de nuestros informantes, identificándolos a partir de su ubicación en el mapeo de actores (C: Sociedad Civil, G: Actor gubernamental, L: Actor local) y al número de entrevista en el orden que fueron recabadas.

\section{Resultados y discusión}

\section{Experiencias de gobernanza hídrica en la política neoliberal en México}

Previo a la implementación del modelo político y económico neoliberal, las políticas de uso y distribución del agua eran una atribución propia del gobierno federal (Aboites, 2004, p. 89). Con la creación de la Comisión Nacional del Agua (CONAGUA) y los diversos cambios de rumbo dados por las administraciones federales en los años 80, surgió un discurso político que definió como prioridad del Estado el cuidado de los recursos hídricos más que la inversión en megaproyectos para irrigación y distribución del vital líquido. Es decir, la política de gestión del agua desde el régimen neoliberal, partió del problema del desperdicio y la contaminación del recurso más que del problema de la falta de acceso al agua como eje de la política de Estado.

El gobierno federal fue desvinculándose de la labor de crear infraestructura para distribución y saneamiento, dejándola a los gobiernos subnacionales a través de reformas que dieron como resultado la "descentralización del agua" (Kloster, 2017, p. 6; Soares, 2007, p. 22). Luego de este proceso, surge la figura jurídica de los OOs locales, los cuales son entidades públicas autónomas o privadas encargadas de la construcción y mejoramiento de infraestructura para distribución y saneamiento del agua y las redes de drenaje, de la administración de recursos financieros para dichas actividades, y del fomento de la participación ciudadana en la cogestión de los recursos hídricos (Kloster, 2017, p. 68). De acuerdo con el directorio de OOs del Centro Virtual de Información sobre el Agua, para 2015 se contabilizaron alrededor de 2,356 OOs distribuidos en toda la república mexicana (VVAA, 2015). El estado de Guerrero registra la operación de ocho OOs de carácter estatal y municipal, el organismo municipal más importante a nivel estatal es la Comisión de Agua Potable y Alcantarillado Municipal de Acapulco (CAPAMA) (VVAA, 2015).

CAPAMA surge en el año de 1977, aún en el marco de una política de gestión federal de los recursos hídricos y como organismo paraestatal dependiente del gobierno estatal. En 1989 CAPAMA se conforma en 
un organismo fiscal autónomo del municipio de Acapulco. Su consejo de administración está integrado por el presidente municipal, el director de salud municipal, el secretario de desarrollo urbano y obras públicas del municipio, el director de desarrollo rural municipal, el coordinador de servicios públicos municipales, el regidor encargado de la comisión de ecología, el regidor encargado de la comisión de servicios públicos municipales, el regidor encargado de la comisión de salud, un representante de CAPASEG y el presidente del consejo consultivo del OO. Más la participación con voz, pero sin voto, de representantes usuarios domésticos, comerciales, industriales y de servicios, siempre y cuando se trate algún tema de su competencia (RICAPAMA, 2008, art. 7). Las funciones de dicho consejo de administración suponen, entre otras, la propuesta de candidatos a director del $\mathrm{OO}$, autorización y aprobación de propuestas generadas por la dirección y la presidencia municipal, aprobación y expedición del reglamento interior de CAPAMA, entre otros (RICAPAMA, 2008, art. 10), en este sentido, la incidencia de dicho órgano tiende hacia la generación de criterios legales y evaluación-validación de acciones directivas.

Otro de los mecanismos de gobernanza es el de los Consejos de Cuenca, que son órganos consultivos con alcance regional encargados de emitir recomendaciones, y planear y evaluar las políticas de gestión del agua en los tres órdenes de gobierno por parte de académicos, organizaciones civiles, usuarios de servicios de los organismos operadores y actores gubernamentales. Los Consejos de Cuenca se constituyen mediante un enfoque de manejo de cuencas, que supone un abordaje integral y sistémico de la gestión de aguas marítimas, fluviales y lagunares que forma parte de una estrategia conocida como Gestión Integral de Recursos Hídricos (GIRH) (Perevochtchikova \& Arellano, 2009, p. 3). En ese sentido, el ámbito de incidencia de los Consejos de Cuenca supone la planeación y evaluación de políticas del agua en los tres órdenes de gobierno (federal, estatal y municipal) dentro de la cuenca determinada.

A diferencia del consejo de administración de CAPAMA, los organismos de gestión comunitaria, no tiene injerencia directa en la gestión. De acuerdo con lo estipulado en los artículos 13, 13Bis, 13Bis 1, 13Bis 2, 13Bis 3 y 13Bis 4 de la Ley Nacional de Aguas, los representantes usuarios y de organizaciones civiles cuentan con voz y voto, e incluso cuentan con una proporción en la representación del Consejo de Cuenca de al menos el 50 por ciento (LAN, 2016, art. 13Bis). Poseen al mismo tiempo, la capacidad de conformarse como Asamblea de Usuarios, con funciones (entre otras) de discutir estrategias y prioridades para la planeación de políticas en torno al uso del recurso hídrico en la cuenca, vigilar el cumplimiento del Plan Hídrico de la Cuenca, nombramiento de vocales y definir posicionamientos sobre coyunturas concretas ante el Consejo de Cuenca (LAN, 2016, art. 
13Bis1, Parágrafo A, incisos 1-5). Entre sus atribuciones, los Consejos de Cuenca impulsan el uso eficiente y sustentable del agua, el "mejoramiento de la cultura del agua", y el conocimiento y difusión de parámetros en materia de recursos hídricos y su gestión (LAN, 2016, art. 13Bis 4).

El Consejo de Cuenca de la Costa Sur de Guerrero ha funcionado desde el año 2000, pero su constitución se confirmó hasta la IV sesión plenaria del Consejo del 25 de agosto de 2012. El Consejo incluye también los consejos auxiliares de subcuenca de los ríos Huacapa-Rio Azul, Rio La Sabana-Laguna de Tres Palos, Laguna de Coyuca-Laguna de Mitla, Río Petatlán, y los comités de Playas Limpias de Acapulco y Zihuatanejo. Dentro de la subcuenca Sabana/Laguna de Tres Palos encontramos experiencias de cogestión basadas en la "gestión comunitaria del agua," que en términos teóricos puede definirse como una forma de gestión del agua como un bien colectivo transgeneracional hereditario, regional, no estatal y administrado por los habitantes de una localidad específica (Escobar, 2017).

De acuerdo con la legislación en materia de aguas en México, existe la figura institucional de los Sistemas de Agua Independientes (SAPI), que son organismos no dependientes de los OOs y que son conformados por Comités de Agua Potable Comunitarios (CAPC). Tan solo en el municipio de Acapulco, de acuerdo con Toribio y López (2015, p. 484), existen alrededor de 60 CAPC que generan un autoabastecimiento ante la falta de cobertura por parte de CAPAMA, habiendo 29 CAPC en la subcuenca del Rio La Sabana. Estas CAPC se rigen bajo el principio de usos y costumbres, donde sus esquemas de participación, distribución de recursos de agua potable y alcantarillado, saneamiento y sanciones, determina cada comunidad en función de diversos mecanismos, como la participación acotada a usuarios con pago regularizado, participación en actividades de tequio o trabajo comunal, etcétera.

\section{Caracterización de la seguridad socioambiental como forma de gobernanza}

A continuación mostramos el mapa semántico derivado del análisis de las citas recuperadas de las entrevistas. Su explicación es el hilo conductor de la presente sección.

Como hemos mencionado en el apartado teórico, al hablar de gobernanza hídrica, consideraremos dos ejes primordiales de análisis: la participación de la ciudadanía en los procesos de toma de decisión, y la incidencia que ésta puede llegar a tener en la gestión del agua. Por un lado, los Consejos de Cuenca pueden otorgar un gran margen de participación de usuarios, otorgándoles capacidad de formar asambleas de usuarios y hacer recomendaciones técnicas a otros órganos gubernamentales o autó- 


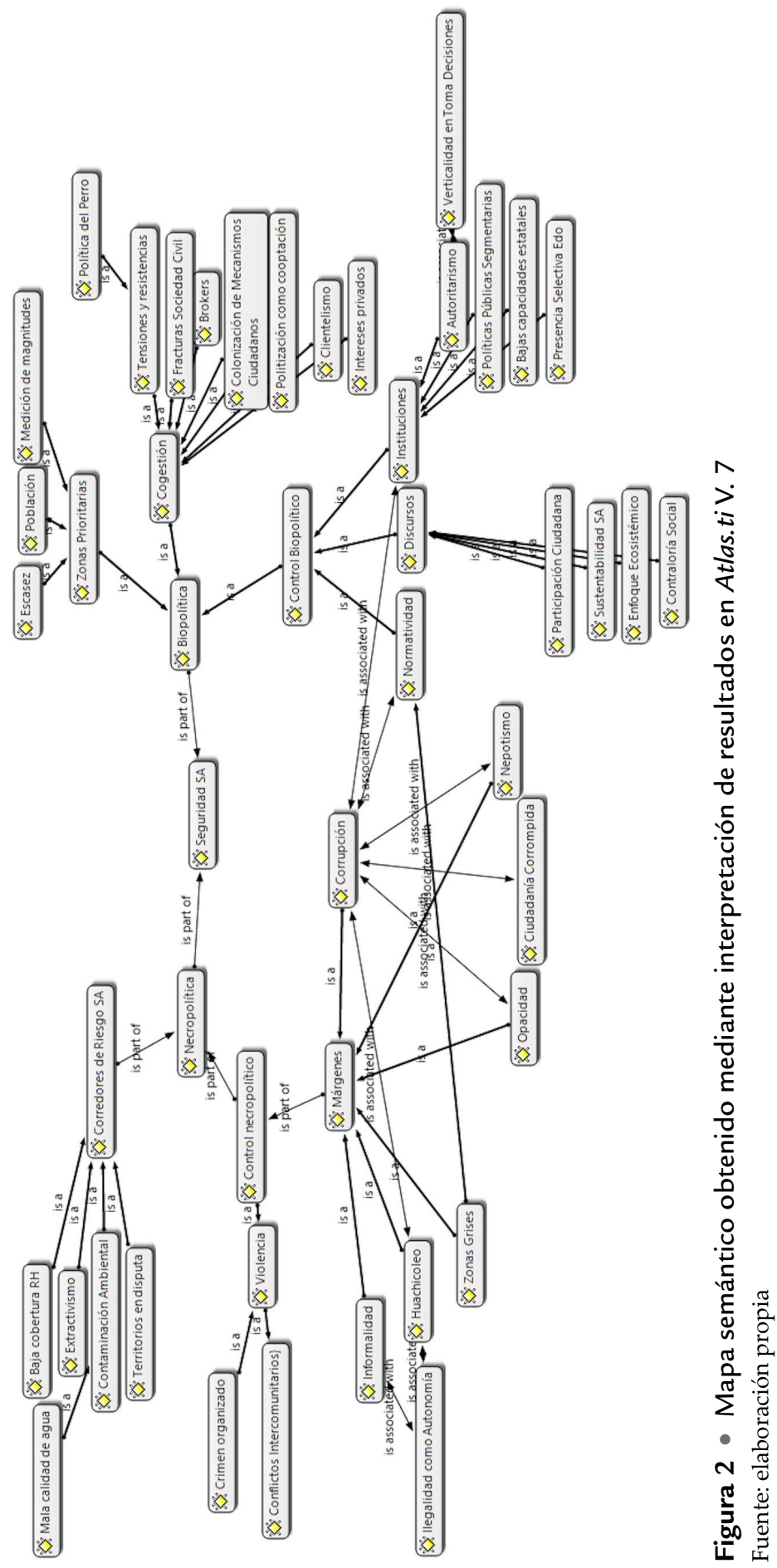


nomos; sin embargo, esto no significa por sí mismo que el usuario, como ciudadano, pueda incidir directamente sobre los recursos hídricos. Por otro lado, el consejo de administración de CAPAMA limita la participación de los usuarios a la voz sin derecho a voto, limitando su capacidad de incidencia sobre los recursos hídricos manejados por el organismo, y reduciendo su participación al mínimo. Finalmente, si bien algunos estudios abordan la relativa autonomía y potenciación de sujetos activos en la gestión comunitaria del agua (Ostrom, 2000), no hay una ley que garantice dicha participación o posibilidad de una equitativa participación, lo cual se define extralegalmente y en función de las condiciones de cooperación comunitaria de cada experiencia.

Ninguna de las experiencias de gobernanza de nuestro análisis, por sí mismas, está orientada hacia un esquema de "mayor participación, mayor incidencia", que pudiera ser garantizado desde un planteamiento orientado al reconocimiento de derechos más que a la securitización. Nuestros referentes empíricos dan como resultado un manejo de la relación sociedad civil-Estado en el aprovechamiento de recursos hídricos desde una perspectiva de "seguridad socioambiental", en la medida que se trata de dispositivos institucionales, legales y procedimentales que buscan proveer a las poblaciones de una percepción positiva de la acción estatal sobre los problemas relativos al manejo de recursos hídricos.

Con la información cualitativa, reconstruiremos la forma en que los actores dan sentido a esta proceso: como "necropolítica" - presencia selectiva del Estado, corrupción, impunidad, abandono de territorios y poblaciones, emergencia de corredores de riesgo socioambiental-, y como "biopolítica" - relativo acceso a la participación ciudadana, construcción de indicadores de medición del problema ajenos a conflictos políticos, contrapesos civiles-.

\section{La gobernanza hídrica como proceso necropolítico}

De acuerdo Mbembé (2011), la necropolítica surge en un momento histórico que puede definirse como un "Estado de excepción", idea que recupera de Carl Schmitt, ${ }^{4}$ donde los actores gubernamentales defienden un Estado amenazado interna o externamente, cuestionando el poder y relatividad del Estado de Derecho (Mbembé, 2011; Schmitt, 2009). Si bien la manifestación más burda de este control mediante la muerte se da a partir del genocidio, esta excepcionalidad-donde el control territorial es imposible da pie a la emergencia del "gobierno privado indirecto". Es decir, en un contexto de desabasto, desinstitucionalización y desterritorialización, el control político de las poblaciones se da a partir de actores privados que operan al margen del Estado-actores criminales, emergencia de econo- 
mías subterráneas e informales-, pero que asumen papeles tradicionalmente atribuidos al mismo, como el cobro de impuestos, la distribución de bienes, la garantía de seguridad pública, etcétera. (Mbembé, 2011).

En cuanto a la excepcionalidad expresada en la apariencia jurídica de participación ciudadana e incidencia no garantizada en el procedimiento directo de la gestión, los actores reportan que no existe necesariamente una consistencia entre los derechos de participación que puedan ser garantizados dentro de los marcos jurídicos de los mecanismos de gobernanza. Esto puede presentarse justo en las reuniones para elaborar tomas de decisión: los espacios físicos de reunión no son aptos para la asistencia de otros ciudadanos, pueden existir formas veladas o directas de amenaza a los ciudadanos que no evidencien la "excepcionalidad", y en términos generales, las recomendaciones dadas por los actores civiles no son necesariamente vinculantes. Sobre los espacios para las sesiones del Consejo de Administración de CAPAMA y el acceso a usuarios dentro de las reuniones, informantes entrevistados nos mostraron algunas imágenes del lugar, una de las cuales mostramos a continuación.

Sobre las recomendaciones del Consejo de Cuenca a instituciones gubernamentales, especialmente una que tiene que ver con la puesta en marcha de acciones para mejorar la calidad del agua en mantos acuíferos subterráneos, un expresidente del Consejo de Cuenca del Rio La SabanaLaguna de Tres Palos nos mencionó que CONAGUA no tenía interés de

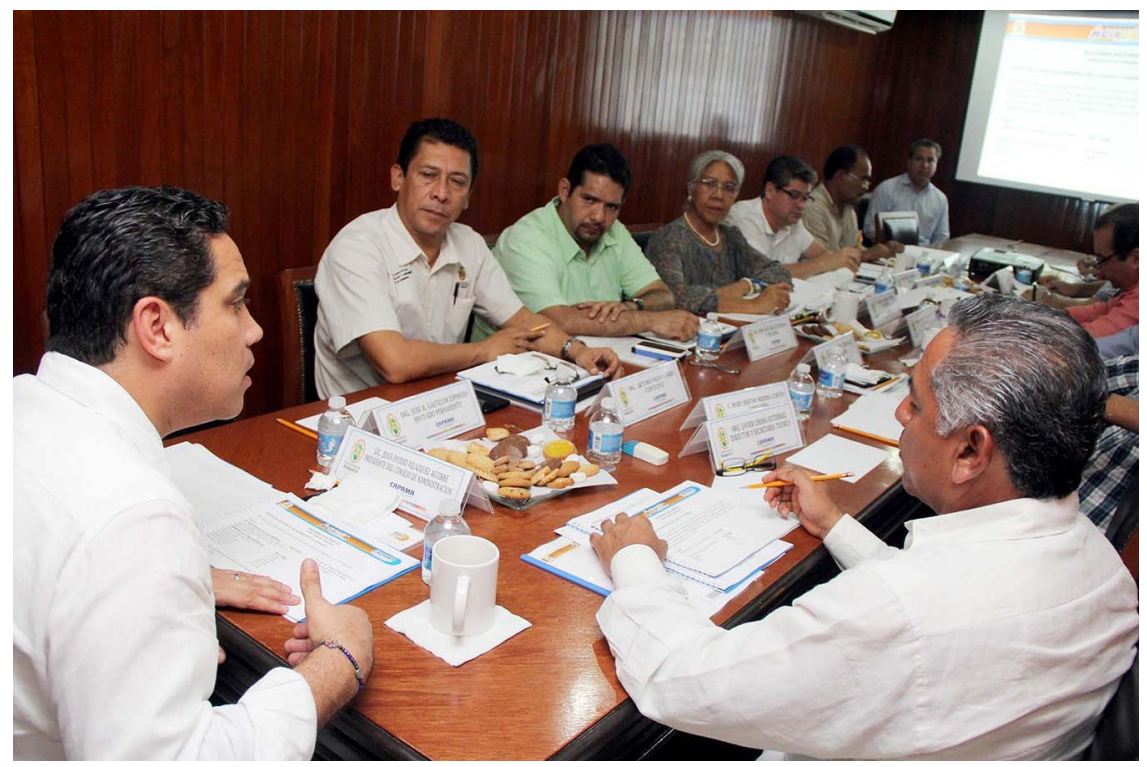

Figura 3 - Sala de reuniones del Consejo de Administración de CAPAMA. Fuente: El Sol de Chilpancingo (2015) 
difundir esta información, y que incluso se le apartó del cargo sin previo aviso, sin algún acta de consejo, e inclusive sin alguna sesión en la que pudiera entregar el cargo (Informante C2).

Y sobre la incidencia de los consejos de cuenca sobre problemáticas directas de acceso al agua, el presidente del Consejo de Cuenca del Rio La Sabana-Laguna de Tres Palos, y un vocal académico del mismo comité, da cuenta de un problema que puede resumirse en que los organismos autónomos generan mayor gasto operativo y menor incidencia sobre las problemáticas que buscan resolver: mencionaron su preocupación por la falta de operatividad de al menos la mitad de organismos de consulta como los consejos de cuenca, y atribuyeron a esta ineficacia los problemas actuales de crisis en la calidad del agua y en la gestión compartida del recurso (Informantes C3 y C4).

La emergencia de problemas como la corrupción, la impunidad y la presencia de legalidades paralelas en la incidencia sobre recursos hídricos también rodea al proceso de gobernanza hídrica. Actores civiles y gubernamentales coinciden en el uso discrecional de recursos por parte de actores políticos sobre los recursos financieros de CAPAMA, pero también ha habido actores dentro de los entornos comunitarios que nos hablan de la venta de vital líquido mediante pipas, que no son reguladas gubernamentalmente ni cuentan con controles de calidad. Sin embargo, son actores gubernamentales los señalados como aquellos que logran sacar ventaja de esta trama de relaciones de ilegalidad y corrupción. Esto no significa que la ilegalidad sea un elemento negativo por sí mismo si seguimos los argumentos de Mbembé o Das y Poole, dado que también se ha asumido que hay actores que, en condiciones de precariedad y falta de acceso a recursos hídricos, han tenido que recurrir al uso de medios no legales para proveerse de agua, sea mediante la compra de pipas, el "huachicoleo" 5 - como le llama el actor $\mathrm{C} 2$ - de tuberías, o la instalación de tuberías no reglamentadas en fuentes subterráneas. De acuerdo con Mbembé (2011), Das y Poole (2008), las formas de sobrevivencia en dichos estados de excepción permiten que los desplazados articulen en la ilegalidad su propia sobrevivencia.

Sobre el saqueo de recursos a CAPAMA, y la impunidad a sus perpetradores, un informante miembro de diversas organizaciones ciudadanas ambientalistas en Acapulco, menciona que son comunes los casos en los cuales los exalcaldes obtienen otros cargos de elección popular (como asientos en el congreso local o federal), con los que logran obtener fuero y evitan cualquier sanción penal por delitos como malversación de fondos públicos, especialmente en el órgano operador de recursos hídricos (Informante C1).

Sobre la trama de relaciones de corrupción entre actores gubernamentales, empleados y empresarios hoteleros-la cual puede entenderse como 
una suerte de "zona gris" de relaciones de ilegalidad orientada hacia la corrupción (Auyero, 2007) - un exfuncionario municipal del área de planeación sostuvo que la sustracción de recursos financieros del organismo operador de agua a modo de "caja chica" era una realidad, y que también podía sostenerse la evasión de cargos por uso de vital líquido a partir de "dobles tomas": una que era medida por CAPAMA, y otra ilegal conectada a la red de distribución del mismo órgano, pero que no era medida para efectuar el cobro (Informante G2). Dicha forma de red de relaciones entre actores legales e ilegales se traduce en lo que Mbembé llama "gobierno privado indirecto", del cual hemos hablado líneas arriba.

Finalmente, este escenario de inseguridad socioambiental, de relaciones desde la ilegalidad y la corrupción para la gestión de recursos hídricos, entre otros factores, han generado lo que denominaremos "corredores de riesgo socioambiental", espacios geográficos impactados ambientalmente, en los que la cotidianidad y las relaciones sociales se definen por la violencia, la segregación y el abandono estatal. Actores civiles y gubernamentales han dado cuenta de la existencia de estas zonas, y la alta peligrosidad que suponen como espacio de vida o de convivencia. El informante C3, por ejemplo, mencionó que hacía 15 años, las condiciones para elaborar estudios científicos dentro de los cauces del río no eran de peligro considerable, como sí lo son ahora. "Ya no se puede compenetrar uno con la gente ahí porque si tú vas y tratas de hacer un trabajo comunitario o algo, lo primero que van a investigar qué vas a hacer, los vas a ayudar o realmente estás viendo a ver qué para denunciar que está pasando algo" (Informante C3).

El informante G2, en el mismo sentido, nos da un panorama del grado de descomposición socioambiental en dichas zonas. Muchas de ellas son asentamientos irregulares de alto riesgo, con problemas de hacinamiento y de alta demanda de servicios públicos que no han sido cubiertos por los gobiernos locales, y que proveen alta rentabilidad electoral obtenida mediante acuerdos clientelares con candidatos a puestos de elección popular o partidos políticos. Además de ser lugares considerados de alta violencia. "Porque como son zonas irregulares donde faltan servicios y todo, entonces, es gente que se dedica a la actividad del trabajo informal, pero también hay ciertos lugares donde no hay actividad económica y se dedican a delinquir, a la delincuencia organizada" (Informante G2). Entre estas actividades, se llega a ofertar la venta no regulada de vital líquido mediante vehículos que trasladan agua obtenida en fuentes subterráneas no registradas ante CONAGUA, por un costo de 60 pesos (aproximadamente 3 USD) por un contenedor doméstico ("tinaco") de 1,000 litros (Informante L8).

El abandono estatal en materia de seguridad pública, la incursión de grupos criminales organizados involucrados en la venta y distribución de agua extraída ilegalmente de mantos subterráneos, y el sentimiento 
generalizado de vulnerabilidad en las comunidades como Kilómetro 30, han generado la emergencia de grupos de autodefensa comunitaria que siguen el mismo mecanismo organizativo de los consejos comunitarios de agua (Informante L8).

\section{La gobernanza hídrica como proceso biopolítico}

La gobernanza hídrica como proceso biopolítico también es producto del proyecto neoliberal, pero tiene objetivos y mecanismos de acción diferentes a los de la gobernanza hídrica necropolítica. La gobernanza hídrica como biopolítica articula una serie de esfuerzos, mecanismos legales, políticas públicas y dispositivos de participación ciudadana que buscan garantizar, en la medida de lo institucionalmente posible, "hacer vivir y dejar morir" (Foucault, 2000, p. 218). Del mismo modo, como proceso biopolítico, la gobernanza hídrica otorga ciertas concesiones a los ciudadanos para que ellos mismos sientan la seguridad de ser quienes inciden sobre las condiciones para que los recursos vitales estén ahí disponibles, con una buena calidad y mediante criterios técnicos orientados por la sostenibilidad.

Ésta no se asume como una satisfacción de derechos históricamente negados a un grupo vulnerable, o a un territorio olvidado por la acción estatal, sino que otorga cierto margen de igualdad de incidencia a los actores que buscan incidir sobre el tema de la distribución, aprovechamiento y mejora de la calidad del agua. El informante C3 menciona que existe participación de usuarios de diversos tipos: "uso industrial, uso agropecuario, uso pecuario, de todo tipo de uso, uso doméstico, e incluso las organizaciones no gubernamentales también participan con uso de asociaciones no gubernamentales ... lo que se busca es que existan voces de todo tipo, sean establecidos o no" (Informante C3).

Discursivamente hablando, la implementación de mecanismos biopolíticos de gobernanza hídrica ocurre bajo una visión eminentemente técnica. Foucault enfatizaba en que el discurso científico y técnico que acompaña la "razón de Estado", ${ }^{6}$ es vital para sostener la legitimidad de dicha toma de decisiones. Conceptos como sustentabilidad, factibilidad técnica de distribución de agua, enfoque ecosistémico, y manejo integral de cuencas, que provienen de discusiones científicas y evidencia empírica, funcionan políticamente para dar cuenta de los límites - reales o autoimpuestos-de la acción del Estado. Con su adopción, se busca generar la percepción en los actores civiles de que los gobernantes: (1) se encuentran comprometidos con la atención a un tema público particular (en este caso, el manejo de recursos hídricos); (2) otorgan a los ciudadanos poder para incidir sobre el problema; y (3) los espacios que recibirán, o no, atención directa del Estado (Informante G1). 
La misma explicación da un trabajador operativo de CAPAMA de la región, para justificar la coexistencia de la distribución comunitaria de agua en Kilómetro 30 y la distribución hecha por su institución de trabajo: "Porque el agua de aquí no sube hasta allá, no sube a las partes altas ... así que las partes altas se mantienen de tanques alternos allá arriba" (Informante L3).

El proceso de cogestión tiende a una mayor participación de los actores civiles, inclusive permitiendo diversas formas de acción colectiva de carácter contencioso que permite apertura del gobierno a la puesta en marcha de propuestas civiles. El informante C2 cita una experiencia de lo que Margaret Keck y Kathryn Sikkink (2000) denominan como "efecto boomerang", es decir, una serie de acciones realizadas para visibilizar una demanda social fuera de la esfera pública local-generalmente acotada por el autoritarismo de los gobernantes subnacionales-, que puede generar adhesiones nacionales e internacionales a la causa de quienes llevan a cabo dicha acción contenciosa. A continuación citamos el evento sucedido en los años 90 del siglo pasado: "Hicimos la colecta, llegaron de Televisa, me entrevistó el corresponsal, salió en 24 horas con Jacobo Zabludovsky, con Abraham Zabludovsky, con Lolita Ayala, y se dio a conocer así un bum. Y una de las palabras dentro de la entrevista, entrevistaron a varios, cuando me entrevistan a mí, nos dice el reportero de televisa que si esto era perjudicial para Acapulco, como había dicho el presidente municipal, le dije que no, que esto era un ejemplo y era un mensaje a todo México, que los guerrerenses y los acapulqueños barremos nuestra casa y que vengan porque va a estar limpia" (Informante C2).

Sin embargo, a pesar de mostrar una mayor libertad de incidencia y participación ciudadana, esto no exime a los actores civiles considerar métodos que pudieran reproducir mecanismos clientelares como propuesta para intervenir en la preservación de la calidad de los recursos hídricos. El informante $\mathrm{C} 2$, por ejemplo, propone en la siguiente cita la implementación de un programa de estímulos a la separación de residuos sólidos a partir del subsidio al precio de la tortilla": "quien separe la basura, quien la lleve al centro de Acopio le dan su bono para que compre su tortilla. ¿cómo?, ¿dónde?, tenemos las tiendas CONASUPO que había en aquel entonces, hay que decirle que la misma tienda CONASUPO, la leche, le dé un peso menos si le llevan la basura" (Informante C2).

La gobernanza hídrica biopolítica busca generar soluciones a ambas problemáticas en territorios específicos, atendiendo problemas por separado, aunque con políticas implementadas al mismo tiempo. Un problema que Foucault detecta en su crítica a Adam Smith y David Ricardo al tratar el tema de la producción de bienes orientada a tres factores-trabajo, tierra y capital-, sin abordar el problema del trabajo en sí. Es decir, Smith y 
Ricardo neutralizaron y naturalizaron el trabajo como un concepto cuantitativo, sin indagar en la crisis humana que supone la precarización laboral o los efectos en la vida cotidiana que supone la pérdida del empleo (Foucault, 2007, pp. 256-257). Desde la gobernanza como biopolítica, sigue siendo viable un proyecto basado principalmente en la puesta en marcha de mecanismos institucionales ciudadanizados, como los que menciona a continuación el informante G2: "A mí me queda claro qué, con buenas decisiones y buenas políticas, y combatiendo la corrupción y la impunidad, se puede llevar a cabo pero la solución: hacer un consejo ciudadanizado" (Informante G2).

\section{Conclusiones}

En la introducción al presente artículo, hemos formulado la pregunta central del mismo: ¿Qué aportes ofrecen los planteamientos teóricos sobre el control y el poder de Foucault y Mbembé para comprender la relación entre actores ciudadanos y gubernamentales en la gestión de recursos hídricos dentro de la subcuenca Río La Sabana-Laguna de Tres Palos? La adopción de un modelo teórico basado en las propuestas de dichos autores para analizar el control hídrico gubernamental, nos permitió identificar la búsqueda de "securitización" del medio ambiente en las experiencias de gobernanza hídrica en la subcuenca Rio La Sabana-Laguna de Tres Palos; proceso que se encuentra en una fase crítica evidenciando la crisis de final de régimen.

Del mismo modo, vemos que las categorías de biopolítica y necropolítica en la gobernanza del agua nos permite ver dos caras del proceso gubernamentalizador de los mecanismos existentes de cogestión hídrica. $\mathrm{Si}$ bien, por un lado, hay una preocupación por medir el problema y generar criterios técnicos que medien entre la necesidad de la población por acceder a recursos hídricos y las capacidades infraestructurales del Estado por proveer del vital líquido, es necesario tener en cuenta que las poblaciones que no cuentan con acceso ni a recursos hídricos ni a la gestión de los mismos hacen lo que pueden con lo que tienen para garantizarse dichos recursos, aún cuando existan medios ilegales o violentos para lograrlo.

Hemos señalado la evidente crisis de la fase de implementación de políticas ambientales orientadas a la mercantilización de los recursos naturales, en la medida que no se han cumplido cabalmente los resultados prometidos de una mayor capacidad del Estado para cumplir con sus objetivos centrales de generar seguridad, estabilidad económica, mayor acceso a recursos naturales y bienestar social (Escalante Gonzalbo, 2015; Mbembé, 2011). Pero el proceso mismo de implementación de la seguri- 
dad socioambiental como estrategia de gobernanza hídrica, evidencia las persistencias de un Estado autoritario y de una ciudadanía fragmentada y temerosa. Si bien los actores pueden recurrir a la ilegalidad y la informalidad como recurso de sobrevivencia ante un Estado omiso a la responsabilidad histórica de proveer de recursos hídricos, esto solo fortalece la desigualdad y la intromisión de actores delincuenciales que mantienen el control del disenso mediante el uso de la violencia. La seguridad socioambiental fortalece así, un "gobierno privado indirecto", y con esa capacidad de "dejar morir" a pesar de no desatar un genocidio directamente, se torna también "necropolítico" (Mbembé, 2011).

Es necesario dar un paso adelante en materia de gestión de recursos hídricos en la subcuenca: pasar de un modelo de seguridad socioambiental, centrado en la generación de una percepción de que los actores ciudadanos son verdaderamente incluidos dentro del proceso de toma de decisiones sobre la gestión del agua, a un modelo de "justicia socioambiental" basado en el reconocimiento de una deuda histórica que el Estado y sus gobernantes han tenido con actores sociales que han sido marginados en para decidir de manera autónoma por sus recursos hídricos. Orientar las políticas públicas en materia socioambiental hacia un enfoque que reivindique derechos, reconozca la deuda estatal con los territorios y poblaciones abandonadas históricamente, y que conduzca a acciones afirmativas y que empoderen a los actores sin causar un relativo abandono como ha venido sucediendo, puede generar otro esquema diferenciado de relaciones locales de cogestión de recursos ambientales. Y no solamente desde el punto de vista de la mejora del acceso a los recursos hídricos, o al incremento de la calidad del agua, sino también la resolución a otras problemáticas transversales a ello: la seguridad, la desigualdad, el acceso a mejores condiciones de vida, etcétera.

Uno de los aspectos que deben ser discutidos a la luz de los presentes resultados tiene una gran relevancia con el debate público actual en México. A saber: la necesidad de fortalecer al Estado en sus capacidades jurídicas, institucionales, técnicas y operativas para incidir de una mejor forma en torno al problema de la crisis socioambiental en nuestro país. Además de la posibilidad de fortalecer a la sociedad civil como un contrapeso público del Estado que garantice una ciudadanía más activa y comprometida con dicha problemática. En el presente estudio hemos detectado paralelamente fortalezas en la práctica de la gobernanza hacia una perspectiva de derechos socioambientales-sectores civiles interesados en la problemática, actores críticos con el estado actual de las relaciones de cogestión de recursos hídricos-; así como muchas debilidades-bajas capacidades institucionales, gubernamentalización del descontento, ilegalidad, corrupción-. También nos queda claro que muchos de estos 
problemas son más comprensibles si les dimensionamos en su contexto sociohistórico, donde el gobierno subnacional ha sido siempre escenario de diversas disputas caciquiles, ciclos de violencia política que han dado pie a la emergencia de grupos guerrilleros o de autodefensa, saqueo de recursos ambientales mediante megaproyectos económicos, etcétera. Dar el paso hacia un esquema de justicia socioambiental supondrá un fortalecimiento tanto de las capacidades institucionales jurídicas del Estado como de la capacidad de acción estratégica de la sociedad civil, pero también deberá ir de por medio la adopción de un proyecto común orientado al combate a la corrupción e impunidad.

ERICK ALFONSO GALÁN CASTRO Catedrático CONACyT comisionado al Centro de Ciencias de Desarrollo Regional de la Universidad Autónoma de Guerrero. Doctor en Investigación en Ciencias Sociales por FLACSO Sede México (2015), Candidato a Investigador Nacional por el Sistema Nacional de Investigadores desde el año 2019. Especializado en temas de estudios sociopolíticos, seguridad y acceso a la justicia, derechos humanos y conflictos socioambientales.

AMÉRICA LIBERTAD RODRíGUEZ HERRERA Profesora-Investigadora Tiempo Completo en el Centro de Ciencias de Desarrollo Regional de la Universidad Autónoma de Guerrero. Doctora en Antropología Social por la Universidad Iberoamericana (2000). Investigadora Nacional Nivel 1 en el Sistema Nacional de Investigadores desde 2015. Miembro del Cuerpo Académico UAGRO-29. Ambiente y Desarrollo. Especializada en temas de diálogo de saberes ambientales, conflictos socioambientales y Desarrollo Regional.

JOSÉ LUIS ROSAS-ACEVEDO Profesor-Investigador Tiempo Completo en el Centro de Ciencias de Desarrollo Regional de la Universidad Autónoma de Guerrero. Doctor en Biotecnología por la Universidad de Colima, Campus Tecomán (2003). Perfil deseable PRODEP desde el año 2007. Especializado en temas como medición de calidad del agua, bio-indicadores, control biológico y macroinvertebrados acuáticos.

\section{NOTES}

1. Kilómetro 30 es la tercera localidad más grande de las 224 que componen el municipio de Acapulco con 6,301 habitantes, después de Acapulco de Juárez (con 673,479 habitantes) y Xaltianguis (con 6,965 habitantes) (SEDESOL, 2018). Es una localidad que posee al mismo tiempo mecanismos de distribución de agua propia del Organismo Operador municipal, y otro de gestión comunitaria.

2. En líneas posteriores nos referimos al concepto de "seguridad" con un enfoque foucaultiano como un mecanismo de control político frente a la escasez del recurso hídrico (o ambiental en general), y no en los términos de "seguri- 
dad hídrica" como la disponibilidad de agua en aceptable cantidad y calidad para su uso y aprovechamiento de Grey \& Sadoff, 2007, o Pacheco-Vega, 2015.

3. Nos referiremos al neoliberalismo como un proyecto político, social y económico, en el que ha sido fundamental la reducción de las atribuciones y capacidades del Estado para el control de los ciudadanos y el mercado (Foucault, 2007).

4. El concepto de "Estado de excepción" de Carl Schmitt es fundamental para entender la implementación de la soberanía del Estado. Es un caso límite, no previsto por el orden jurídico vigente, donde peligra la existencia del Estado mismo, y con ello, se encuentra amenazado el orden social (Schmitt, 2009, pp. 13-14).

5. "Huachicoleo" referido a la extracción clandestina de hidrocarburos. El actor entrevistado hace un uso metafórico del término.

6. La "razón de Estado" es lo que Foucault llama "el arte del buen gobierno". Son los mecanismos racionalizados, y moralmente aceptados dentro de un corte histórico-episteme-, de la acción política que caracterizan a la biopolítica. (Foucault, 2007, p. 19)

7. Kristen Appendini (1992) ha demostrado que los programas de subsidio a la tortilla en México - tortibonos - fueron un eficaz instrumento de control político territorial en zonas rurales y urbanas de este país, generando relaciones clientelares y fortaleciendo el poder de líderes políticos barriales.

\section{REFERENCIAS}

Aboites, L. (2004). De bastión a amenaza: Agua, políticas públicas y cambio institucional en México, 1947-2001. En B. Graizbord, \& J. Arroyo-Alejandre (Coords.), El futuro del agua en México (pp. 89-114.). México: COLMEX/U. de G./UCLA/Juan Pablos Editores.

Aguilar Villanueva, L. (2016). Democracia, gobernabilidad y gobernanza. México: INE. Appendini, K. (1992). De la milpa a los tortibonos: La reestructuración de la política alimentaria en México. México: El Colegio de México.

Auyero, J. (2007). La zona gris: Violencia colectiva y politica partidaria en la Argentina Contemporánea. Buenos Aires: Siglo XXI.

Castro, J. E., Kloster, K., \& Torregrosa, M. L. (2004). Ciudadanía y gobernabilidad en México: El caso de la conflictividad y la participación social en torno a la gestión del agua. En B. Jiménez, \& L. Marín (Coords.), El agua en México vista desde la Academia (pp. 339-370). México, AMC.

Cohen, J., \& Arato, A. (2001). Sociedad civil y teoría política. México: FCE.

Das, V., \& Poole, D. (2008). El Estado y sus márgenes. Cuadernos de Antropología Social 27, 19-52.

Donoso, J. (2017). Reporte de investigación No. 2. Evaluación del proceso de implementación del Programa Nacional de Prevención del Delito (PRONAPRED) en Acapulco y su impacto en la reducción de violencia. Acapulco: IIEPA-IMA/UAGro.

El Sol de Chilpancingo. (2015, 10 diciembre). Alcalde 'toma' protesta a integrantes del Consejo de Administración de CAPAMA. Descargado de http://elsoldechilpan 
cingo.mx/2015/12/10/alcalde-toma-protesta-a-integrantes-del-consejo-de-ad ministracion-de-capama/.

Escalante Gonzalbo, F. (2015). Historia mínima del neoliberalismo. México: El Colegio de México.

Escobar, C. (2017). Las presidencias de comunidad en tres municipios del estado de Tlaxcala y su relación con la gestión comunitaria de agua. En M. Torregrosa (Coord.), El conflicto del agua: Política, gestión, resistencia y demanda social (pp. 277-296). México: FLACSO.

Falomir Archambault, E. (2011). Introducción. En A. Mbembé, Necropolítica. Madrid: Melusina.

Foucault, M. (2000). Defender la sociedad. Buenos Aires: FCE.

Foucault, M. (2007). El nacimiento de la biopolítica. Buenos Aires: FCE.

Foucault, M. (2013). La inquietud por la verdad: Escritos sobre la sexualidad y el sujeto. Buenos Aires: Siglo XXI

Foucault, M. (2018). Seguridad, territorio, población. Buenos Aires: FCE.

González, J., Quiterio, A., Niño, N., Reyes, M. y Torres, G. (2012): “Evaluación ambiental territorial de la cuenca Río La Sabana - Laguna de Tres Palos". En Sampedro, M. y González, J. (Aut.): Calidad Ambiental y Desarrollo Sustentable. Indicadores. Chilpancingo: UAGro.

Grey, D., \& Sadoff, C. (2007). Sink or swim? Water security for growth and development. Water Policy 9(6), 545-571.

Illades, C. (2010). Guerrero: Una historia breve. México: FCE/El Colegio de México.

Kloster, K. (2017). Gobierno y lucha por el territorio político del agua en México.

En M. Torregrosa (Coord.), El conflicto del agua: Política, gestión, resistencia y demanda social (pp. 61-83). México: FLACSO.

Keck, M., \& Sikkink, K. (2000). Activistas sin fronteras. México: Siglo XXI.

Ley de Aguas Nacionales (LAN). (2016, 24 marzo). Ciudad de México.

Mbembé, A. (2011). Necropolítica. Madrid: Melusina.

Mussetta, P. (2009). Participación y gobernanza. El modelo de gobierno del agua en México. Espacios Públicos. XII(25), 66-84.

Ostrom, E. (2000). El gobierno de los bienes comunes. México: FCE.

Pacheco-Vega, R. (2015). Agua embotellada en México: De la privatización del suministro a la mercantilización de los recursos hídricos. Espiral, estudios sobre Estado y Sociedad XXII(63), 221-263.

Perevotchikova, M., \& Arellano, J. (2009). Gestión de cuencas hidrográficas: experiencias y desafíos en México y Rusia. México: COLMEX/CONAGUA/SEMARNAT.

Reglamento interior de la Comisión de Agua Potable y Alcantarillado del Municipio de Acapulco (RICAPAMA). Acapulco, Guerrero, 8 de agosto de 2008.

Saldaña, E. y Pérez, S. (2015): Torrente y su mal manejo. Acapulco: UACA-UAGro.

Sánchez Infante, A. (2018). Aspectos para lograr la sustentabilidad en la zona norte de la Laguna de Tres Palos. En A. Sánchez Infante, \& A. Rosas Acevedo (Coords.), Medio ambiente, sociedad y turismo (pp. 7-26). México: Porrúa.

Sánchez Infante, A. y Rosas Acevedo, A. (Coords.) (2018). Medio ambiente, sociedad y turismo. México: Porrúa. Schmitt, C. (2009). Teología política. Madrid: Trotta.

SEDESOL. (2018). Municipio de Acapulco de Juárez: Catálogo de localidades del sistema de 
apoyo para la planeación del PDZP. Descargado de: http://www.microrregiones .gob.mx/catloc/LocdeMun.aspx?tipo=clave\&campo=loc\&ent=12\&mun=001.

Soares, D. (2007). Crónica de un fracaso anunciado: La descentralización en la gestión del agua potable en México. Agricultura, Sociedad y Desarrollo 1(4), 19-37.

Triana, J. 2017. Diagnóstico integral de las instituciones de seguridad y de justicia en Acapulco. Revisado en http://iiepa.uagro.mx/inicio/images/pdf/JORGETRIANA .pdf [25 de abril de 2019]

Toribio, G. y López, A. (2015). La perspectiva de agua en Guerrero, limitaciones y retos para el desarrollo. Revista Mexicana de Ciencias Agrícolas. I(1), 479-486.

VVAA. (2015). Directorio de organismos operadores de agua potable y alcantarillado. México: Fondo para la Comunicación y la Educación Ambiental A.C.

Water governance as socio-environmental securitization in La Sabana-Tres Palos sub-basin, Acapulco

\title{
Erick Alfonso Galán Castro, América Libertad Rodríguez Herrera \\ \& José Luis Rosas-Acevedo
}

\begin{abstract}
This article analyzes three types of water governance in the sub-basin of La Sabana River-Tres Palos Lagoon from the perspective of Michel Foucault's governmentality. These processes-including the board of directors of the Metropolitan Water Operator Organism called the Drinking Water and Sewage Commission of the Municipality of Acapulco (CAPAMA), the Basin Council of the La Sabana River-Laguna de Tres Palos Lagoon, and an experience in community water management in the town of Kilómetro 30, in the same municipality of Acapulco-are addressed through analysis based on the following question: How is the relationship between citizens and officials for water management in the Acapulco region governed? The actors perceive a greater tendency for political control than democratization in decision-making.
\end{abstract}

Keywords: Basin territories, citizenship, governmentality, illegalities, water Resources

\section{Article 03: La gouvernance de l'eau comme sécurisation socio- environnementale dans le sous-bassin de La Sabana-Tres Palos à Acapulco}

\author{
Erick Alfonso Galán Castro, América Libertad Rodríguez Herrera \\ \& José Luis Rosas-Acevedo
}

Résumé: Cet article analyse trois types de gouvernance de l'eau dans le sous-bassin de la rivière La Sabana-Laguna de tres Palos du point de vue de la gouvernementalité de Michel Foucault. Ces instances -le conseil d'administration de l'organisme métropolitain de l'exploitant de l'eau ap- 
pelé Commission de l'eau potable et des égouts de la municipalité d'Acapulco (CAPAMA), le conseil du bassin du Río la Sabana-Laguna de Tres Palos, et une expérience dans la gestion communautaire de l'eau dans la localité du Kilometro 30, dans la même municipalité d'Acapulco-, sont observées à partir de la question suivante: comment la relation entre les citoyens et les responsables de la gestion de l'eau est-elle gouvernementalisée dans cette région? Il en ressort qu'en ce qui concerne la prise de décision, les acteurs perçoivent davantage une tendance au contrôle politique qu'à la démocratisation.

Mots-clés: Citoyenneté, gouvernementalité, illégalités, ressources en eau, bassin versant, territoire. 